[3] Guyatt G, Oxman A, Vist G, et al. GRADE: an emerging consensus on rating quality of evidence and strength of recommendations. BMJ 2008;336:924-6. Disclosure of Interest: None declared

DOI: 10.1136/annrheumdis-2017-eular.2085

\section{THU0562 INFLAMMATORY JOINT DISEASE TRIGGERED BY IMMUNE CHECKPOINT INHIBITORS}

L. Tucker, S. Sacks, H. Al-Mossawi. Rheumatology, Oxford University Hospitals NHS Foundation Trust, OXFORD, United Kingdom

Background: Immune checkpoint modulation has changed the cancer therapy landscape in recent years [1]. Randomised controlled trials now demonstrate superiority to standard chemotherapy in squamous cell lung cancer, renal cell cancer [2], squamous cell head and neck cancer [3] and malignant melanoma [4]. However, blockade of these checkpoints results in a multitude of autoimmune sequelae including joint disease. As these therapies become first-line in oncology, they will likely place a significant burden on rheumatology services.

Objectives: To establish the prevalence of rheumatological manifestations of checkpoint blockade in a non-trial standard care setting.

Methods: All patients treated with Nivolumab or Ipilimumab (as single agent or in combination) in a standard care setting within a single centre were included and the number of suspected cases with rheumatological manifestations identified from the case records.

Results: 75 patients have been treated with Nivolumab single agent and 12 with combination Nivolumab plus Ipilimumab in Oxford over the last 15 months. Within that cohort, there were 7 suspected cases of joint disease giving a potential prevalence of $8 \%$. Of those 7,2 had confirmed disease requiring therapy, both had received Nivolumab/lpilimumab combination. Both cases were rheumatoid factor and CCP negative. One case responded to prednisolone only, while the second case was refractory to steroids, methotrexate and infliximab but responded to IL-6 blockade with Tocilizumab.

Conclusions: We observed an approximate $8 \%$ prevalence of checkpoint mediated autoimmune joint disease within a relatively short follow up period of 15 months. The longer-term prevalence may be higher. Both patients requiring therapy had been exposed to Ipilimumab suggesting the inflammatory joint toxicity with this agent may be higher than that caused by Nivolumab.

Checkpoint immunotherapy is set to become the first-line standard of care in a number of cancers. This will lead to an increased demand for rheumatology services. The rheumatology community needs to develop strategies and trials in order to classify and treat these patients appropriately.

References:

[1] Mellman I, Coukos G, Dranoff G. Cancer immunotherapy comes of age. Nature. 2011; 480:480-9.

[2] Motzer RJ, Escudier B, McDermott DF, et al. Nivolumab versus Everolimus in Advanced Renal-Cell Carcinoma. NEJM. 2015; 373:1803-1813.

[3] Ferris RL, Blumenschein G, Fayette J, et al. Nivolumab for Recurrent Squamous-Cell Carcinoma of the Head and Neck. NEJM. 2016; 375:18561867.

[4] Larkin J, Chiaron-Sileni V, Gonzalez R et al. Combined Nivolumab and Ipilimumab or Monotherapy in Untreated Melanoma. N Engl J Med. 2015; 373:23-34.

Disclosure of Interest: None declared

DOI: 10.1136/annrheumdis-2017-eular.3875

\section{THU0563 THE IMMUNOMODULATION EFFECT OF BM-MSCS ON THE INFLAMMATORY CHEMOKINES OF RATS WITH COLLAGEN TYPE II INDUCED ARTHRITIS}

J. Fan, L. Zhang, D. Ma, G. Zhang, K. Xu, J. Gao. Rheumatology, Shanxi DaYi Hospital, Shanxi Academy of Medical Sciences, Taiyuan, China

Background: Rheumatoid arthritis (RA) is an autoimunme disease. The DMARDs is mian treatment but side effects are very high. Bone marrow mesenchymal stem cells (MSCs) have immunoregulatory function so it can be used to treat RA. This study was designed to investigate the effect of BMSCs on chemokines in CIA rats.

Objectives: To observe the immunomodulation effects of BM-MSCs on the Inflammatory Chemokines of rats with collagen-induced arthritis (CIA).

Methods: Rats MSCs were isolated and expanded from bone marrow cells by density gradient centrifugation and adhering to the culture plastic bottle, and the phenotypes were assessed by flow cytometry. We established collagen induced arthritis rats model. A total of six groups. The MSCs treatment groups contain the early and advanced stages. Meanwhile, there are normal control and CIA control group, The latter also contains the early and advanced stages. Also,there is the positive control group of MTX, by intraperitoneal injection of a small dose of MTX $(0.9 \mathrm{mg} / \mathrm{kg} / \mathrm{w})$, for six weeks. $1 \times 10^{6} / \mathrm{kg} \mathrm{BM}$-MSCs were implanted through tail vein to MSCs treatment groups, model control groups with equal amounts of saline served as controls.At forty two day,stream multi-factor detection technique were used to detect the level of RANTES, MCP-1 and IP-10 in serum of CIA rats.

Results: The level of RANTES, AMCP-1, AIP-10 was higher in CIA control group than normal group $(P<0.05)$; After MTX treatment, these factors level were significantly difference than the CIA control group $(P<0.05)$. These factors level were lower in early BMSCs treatment groups than early CIA control groups $(\mathrm{P}<0.05)$, while the late MSCs treatment group versus the later CIA control group showed no statistically significant difference $(P>0.05)$. The early and late MSCs treatment group versus the MTX treatment group showed no statistically significant difference $(P>0.05)$.

Conclusions: In this study MSCs has shown significant immune modulatory effects. It down regulates the level of Inflammatory Chemokines in CIA rats. The early treatment group is more effective than the late treatment group.

References:

[1] Stuad JM,Cremer MA,Townes AS,et a1.Type II collagen induced arthritis in rats: passive transfer with serum and evidence that $\lg G$ antieollagen antibodies can cause arthritis.J Exp Med,1982,155:I-16

[2] Brahn E, Peacock DJ, Banquerigo ML. Suppression of collagen-induced arthritis by combination cyclosporin $\mathrm{A}$ and methotrexate therapy. Arthritis Rheum.1998,34 (10):1282-1288.

[3] Dominici M, Le Blanc K, Mueller I, et al. Minimal criteria for defining multipotent mesenchymal stromal cells. The International Society for Cellular Therapy position statement. Cytotherapy. 2006;8(4):315-317.

[4] Park MJ, Kwok SK, Lee SH, et al.Adipose tissue-derived mesenchymal stem cells induce expansion of Interleukin-10 producing regulatory B cells and ameliorate autoimmunity in a murine model of systemic lupus erythematosus. Cho Cell Transplant. 2014 Dec 12. [Epub ahead of print].

[5] Kehoe O, Cartwright A, Askari A,et al.Intra-articular injection of mesenchymal stem cells leads to reduced inflammation and cartilage damage in murine antigen-induced arthritis. J Transl Med. 2014,12:157.

Acknowledgements: Mesenchymal stem cell;Arthritis,rheumatoid; Collagen II induced arthritis (CIA);Inflammatory chemokine.

Disclosure of Interest: None declared

DOI: 10.1136/annrheumdis-2017-eular.3470

\section{THU0564 IGG4-RELATED DISEASE IN THE MALTESE POPULATION}

S. Chetcuti Zammit ${ }^{1}$, M. Rogers ${ }^{2}$, P. Ellul ${ }^{1} .{ }^{1}$ Gastroenterology; ${ }^{2}$ Rheumatology, Mater Dei Hospital, Msida, Malta

Background: IgG4-related disease (IgG4-RD) is an increasingly recognised immune-mediated fibro-inflammatory condition, most commonly affecting the pancreas.

Objectives: The aim of our study was to determine the incidence, organ involvement and outcome of IgG4-RD in the Maltese population.

Methods: The case notes of all patients who had their lgG4 level checked between 2008 and 2015 at Mater Dei Hospital were reviewed. Patients who had their IgG4 level checked inappropriately were excluded. Patients were then stratified according to the "Comprehensive diagnostic criteria for IgG4-related disease" [1].

Results: 25 patients were included in this retrospective, observational study. $60 \%$ $(n=15)$ of patients were males and mean age was 70 years $(S D \pm 64.8)$. The mean lgG4 level was $0.7159 \mathrm{~g} / \mathrm{L}$ (SD \pm 0.851 ) (normal range 0.052-1.250g/L).

Organs involved included: pancreas $(n=15)$, biliary tree $(n=3)$, salivary glands $(n=1)$, lymph nodes $(n=8)$, lungs $(n=4)$, thyroid gland $(n=2)$, bone $(n=1)$, heart $(n=1)$, sinuses $(n=2) .36 \%$ (9 patients) had involvement of more than one organ. Blood tests at time of diagnosis demonstrated a raised mean ESR $(65.7 \mathrm{~mm} 1 \mathrm{st}$ $\mathrm{Hr} ; \mathrm{SD} \pm 36.1)$ and a raised mean $\mathrm{CRP}(143 \mathrm{mg} / \mathrm{L} \mathrm{SD} \pm 112)$. White cell count was mostly normal (mean $11.7 \times 10^{9} / \mathrm{L} \mathrm{SD} \pm 4.91$ ).

$92 \%$ of patients $(n=23)$ had organ swelling on radiological testing. $32 \%(n=8)$ of patients had raised lgG4 levels. A histological diagnosis was obtained in $8 \%(n=2)$ of patients presenting with cervical lymphadenopathy and sinusitis respectively. According to the "Comprehensive diagnostic criteria for lgG4-RD"1, the diagnosis was definite in $4 \%(n=1)$, possible in $28 \%(n=7)$ and probable in $4 \%(n=1)$ of patients. $64 \%$ ( $n=16)$ only had organ swelling on imaging studies, satisfying 1 criterion.

One patient was diagnosed with malignancy during the course of disease. Patients with hepatobiliary and pancreatic involvement underwent ERCP and stenting. Patients who developed pancreatic pseudocyst as a complication of pancreatitis underwent drainage. One patient $(4 \%)$ developed pancreatic insufficiency and $32 \%(n=8)$ experienced recurrent pancreatitis requiring hospital admission. Four patients $(16 \%)$ passed away.

Conclusions: In concordance with current literature, our study has shown that IgG4-RD in the Maltese population is a rare disorder affecting mainly elderly males. The commonest organs involved were the pancreas and lymph nodes. The inflammatory markers ESR and CRP were always raised, thus acting as possible biomarkers for monitoring of disease activity.

References:

[1] Umehara H, Okazaki K, Masaki Y, Kawano M, Yamamoto M, Saeki T et al. Comprehensive diagnostic criteria for lgG4-related disease (IgG4-RD), 2011. Mod Rheumatol. 2012 Feb;22(1):21-30.

Disclosure of Interest: None declared

DOI: 10.1136/annrheumdis-2017-eular.3713 